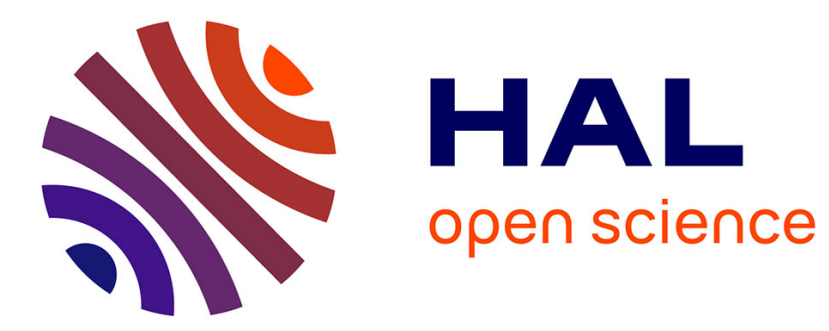

\title{
Matériaux électrostrictifs pour actuateurs
}

\author{
L. Eyraud, P. Eyraud, P. Gonnard, M. Troccaz
}

\section{To cite this version:}

L. Eyraud, P. Eyraud, P. Gonnard, M. Troccaz. Matériaux électrostrictifs pour actuateurs. Revue de Physique Appliquée, 1988, 23 (5), pp.879-889. 10.1051/rphysap:01988002305087900 . jpa-00245891

\section{HAL Id: jpa-00245891 https://hal.science/jpa-00245891}

Submitted on 1 Jan 1988

HAL is a multi-disciplinary open access archive for the deposit and dissemination of scientific research documents, whether they are published or not. The documents may come from teaching and research institutions in France or abroad, or from public or private research centers.
L'archive ouverte pluridisciplinaire HAL, est destinée au dépôt et à la diffusion de documents scientifiques de niveau recherche, publiés ou non, émanant des établissements d'enseignement et de recherche français ou étrangers, des laboratoires publics ou privés. 
Classification

Physics Abstracts

$77.60-81.20 \mathrm{~L}-81.40$

\title{
Matériaux électrostrictifs pour actuateurs
}

\author{
L. Eyraud, P. Eyraud, P. Gonnard et M. Troccaz \\ Laboratoire de Génie Electrique et Ferroélectricité, INSA, 20 avenue Albert-Einstein, Bâtiment 504, 69621 \\ Villeurbanne Cedex, France
}

(Reçu le 10 août 1987, révisé le 4 janvier 1988, accepté le 19 janvier 1988)

\begin{abstract}
Résumé. - Les matériaux électrostrictifs sous forme de céramique, à fort coefficient d'électrostriction, sont très recherchés pour certains transducteurs de déplacement. La recherche de matériaux aussi performants que le PMN-PT, matériau le plus utilisé actuellement, nous a amenés à étudier une composition du type PZT fortement substituée par des ions $\mathrm{Sr}-\mathrm{Ba}$ et de préparation plus aisée. Deux méthodes de fabrication sont utilisées : la voie solide classique et la coprécipitation qui permet d'obtenir des céramiques plus denses et de granulométrie plus régulière On montre dans cette étude que les conditions de frittage ont une influence à la fois sur l'amplitude et l'hystérésis de la déformation. Une comparaison des constantes électrostrictives du composé choisi avec des céramiques PMN-PT et PLZT est donnée.
\end{abstract}

\begin{abstract}
Electrostrictive ceramic materials with a large electrostriction coefficient are intensively investigated for some displacement transducers. In order to obtain materials as good as PMN-PT, a material newly developed but not very easy to prepare, a solid solution of PZT type widely substituted with $\mathrm{Sr}$-Ba ions has been studied. Two methods of preparation were used : a conventional solid one and a coprecipitation one which allows the fabrication of ceramics with a better density and an uniform grain size. In this paper it is shown that the sintering conditions have an influence both on the magnitude and on the hysteresis of the field induced strain. A comparison of the electrostrictive constants of the PZT selected composition with PMN-PT and PLZT ceramics is given.
\end{abstract}

\section{Introduction.}

L'électrostriction est un phénomène qui donne naissance à une déformation proportionnelle au carré du champ électrique appliqué. Il est observé dans tous les diélectriques amorphes ou cristallins [1]. Dans les cristaux ou céramiques piézoélectriques, la déformation produite par un champ électrique appliqué est due à la superposition des effets électrostrictifs et piézoélectriques. Le phénomène d'électrostriction étant en général beaucoup plus faible que l'effet piézoélectrique, les résonateurs, transducteurs et actuateurs ont jusqu'à ces dernières années utilisé les céramiques piézoélectriques malgré certains inconvénients majeurs tels qu'une hystérésis importante et un effet de vieillissement non négligeable.

De récentes études sur les matériaux électrostrictifs ont changé la situation, en particulier pour les raisons suivantes :

- les dispositifs à électrostriction ont une bonne reproductibilité sous des champs électriques cycliques
- certains électrostrictifs peuvent être piézoactifs sous champ électrique continu tout en conservant une faible hystérésis et un faible vieillissement sous champ électrique variable

- certains matériaux électrostrictifs présentent des déformations induites par le champ électrique comparables ou supérieures à celles obtenues avec des céramiques ferroélectriques.

Les compositions les plus prometteuses sont celles qui ont une transition de phase diffuse à une température voisine de la température ambiante. En particulier les compositions à base de $\mathrm{Pb}\left(\mathrm{Mg}_{1 / 3} \mathrm{Nb}_{2 / 3}\right) \mathrm{O}_{3}(\mathrm{PMN})$ ont été beaucoup étudiées [2-6].

Par exemple, une déformation relative de $0,1 \%$ pour un champ appliqué de $1500 \mathrm{~V} / \mathrm{mm}$, sans hystérésis montée-descente, peut être obtenue avec ce type de matériau.

Les applications et la commercialisation de ces matériaux se sont déjà développées au Japon et aux U.S.A. dans les domaines suivants par ordre d'importance [7] : 
- jet d'encre

- positionneur de tête magnétique

- dispositifs d'optique adaptative (miroirs déformables)

- tête d'impression

- moteur à ultrason

— réseaux d'imagerie apodisés.

L'objet de la présente note est la recherche de matériaux céramiques frittables à fort coefficient d'électrostriction utilisables comme actuateurs.

On désigne sous le nom d'actuateur piézoélectrique un système constitué par un empilement de céramiques connectées en parallèle, comme indiqué figure 1.

Par rapport aux actuateurs électromagnétiques généralement utilisés, qui peuvent produire des déplacements plus importants, ce nouveau type d'actuateurs présente cependant les avantages suivants : moindre consommation d'énergie, moindre production de chaleur, absence de bruit. Ils offrent en outre l'intérêt d'avoir un encombrement et un poids faibles et d'être résistants aux chocs et aux vibrations.

\section{Electrostriction dans les matériaux ferroélectri-} ques.

\subsection{COEFFICIENTS D'ÉLECTROSTRICTION $Q$. -} Soient $x_{i}$ les déformations élastiques $(x=\Delta \ell / \ell)$ et $P_{i}$ les polarisations dans les différentes directions d'un cristal.

On peut définir pour un cristal libre de contraintes trois coefficients d'électrostriction $Q$ reliant les déformations au carré des polarisations sous la forme matricielle :

$$
\begin{aligned}
\left|\begin{array}{l}
x_{1} \\
x_{2} \\
x_{3} \\
x_{4} \\
x_{5} \\
x_{6}
\end{array}\right|=\left|\begin{array}{llllll}
Q_{11} & Q_{12} & Q_{12} & 0 & 0 & 0 \\
Q_{12} & Q_{11} & Q_{12} & 0 & 0 & 0 \\
Q_{12} & Q_{12} & Q_{11} & 0 & 0 & 0 \\
0 & 0 & 0 & Q_{44} & 0 & 0 \\
0 & 0 & 0 & 0 & Q_{44} & 0 \\
0 & 0 & 0 & 0 & 0 & Q_{44}
\end{array}\right| \times \\
\left|\begin{array}{l}
P_{1}^{2} \\
P_{2}^{2} \\
P_{3}^{2} \\
P_{2} P_{3} \\
P_{3} P_{1} \\
P_{1} P_{2}
\end{array}\right| \text { Unité de } Q: \mathrm{m}^{4} C^{-2}
\end{aligned}
$$

$Q_{11}=$ coefficient d'électrostriction longitudinal

$Q_{12}=$ coefficient d'électrostriction transverse

$Q_{44}=$ coefficient d'électrostriction de cisaillement

Pour une céramique, la polarisation induite ou spontanée correspond uniquement à une seule direction; on a $P_{2}=P_{3}=0$.

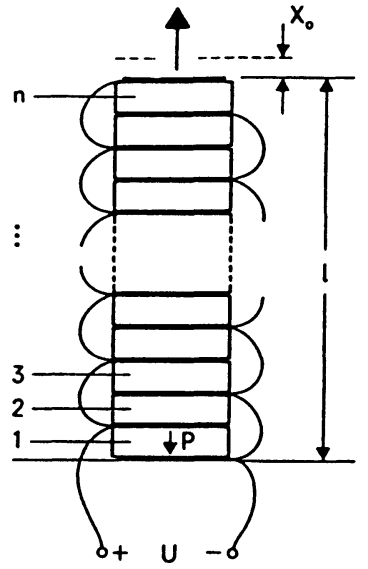

Fig. 1. - Schéma de principe d'un actuateur.

[Schematic representation of an actuator.]

On peut définir un coefficient d'électrostriction hydrostatique $Q_{\mathrm{h}}$ reliant la variation de volume $\Delta V / V$ au carré de la polarisation

$$
\begin{aligned}
\frac{\Delta V}{V} & =Q_{\mathrm{h}} P_{1}^{2} \\
Q_{\mathrm{h}} & =Q_{11}+2 Q_{12} .
\end{aligned}
$$

D'après [5], $Q_{\mathrm{h}}$ varie comme le carré du coefficient de dilatation thermique $\alpha$ et le produit $Q_{\mathrm{h}} C(C=$ Constante de Curie) resterait constant pour les divers matériaux électrostrictifs.

2.2 COEFFICIENTS D'ÉLECTROSTRICTION $M$. Comme précédemment, on peut définir des coefficients d'électrostriction reliant les déformations au champ électrique appliqué par la relation:

$$
x=M E^{2} \text {. }
$$

Si les variations de $P_{1}$ en fonction de $E_{1}$ sont linéaires (polarisation induite), on peut écrire (Fig. 2, traits pointillés) :

d'où

$$
P_{1}=\varepsilon E_{1}
$$

$$
x_{1}=Q_{11} P_{1}^{2}=Q_{11} \varepsilon^{2} E_{1}^{2}
$$

donc

$$
M_{11}=Q_{11} \varepsilon^{2}
$$

où $\varepsilon=\varepsilon_{0} \varepsilon_{33 \mathrm{r}}$ est la constante diélectrique statique calculée à partir de la courbe $P=f(E)$.

De même :

$$
M_{12}=Q_{12} \varepsilon^{2}
$$

Les coefficients $M$ représentent un facteur de mérite du matériau.

Lorsque la courbe $P_{1}=f\left(E_{1}\right)$ est un cycle d'hystérésis (Fig. 2, traits pleins) on peut retracer pour chaque valeur de $E_{1}$ la courbe $x_{1}=f\left(P_{1}\right)$ et en déduire la valeur de $Q_{11}$. 
Tableau I. - Tableau synoptique de la préparation des matériaux par voie solide.

[Flow chart of the material preparation through the solid process.]
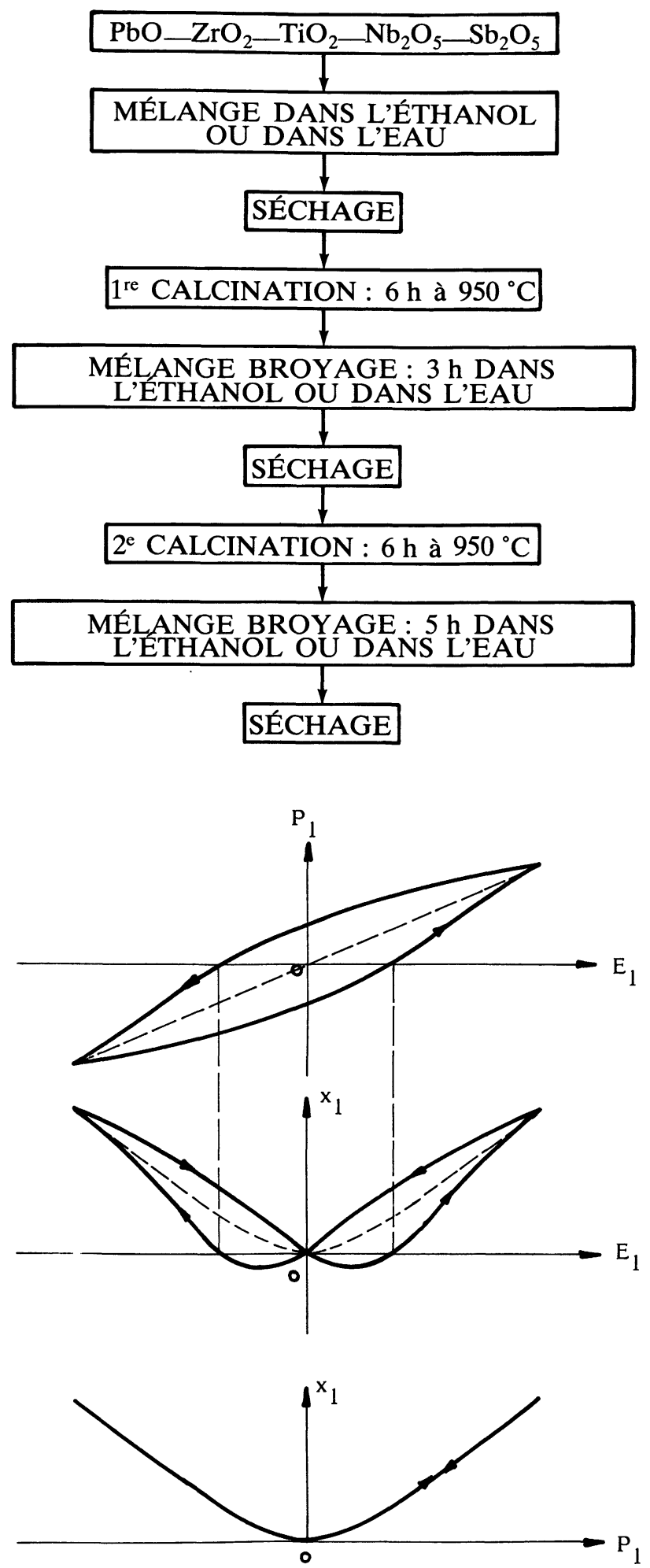

Fig. 2. - Polarisation et déformation en fonction du champ électrique pour un matériau linéaire et non linéaire.

[Polarization and strain versus electric field for a linear and a non linear material.]
2.3 CARACTÉRISTIQueS DES MATÉRIAUX ÉXISTANTS. - Parmi les divers matériaux électrostrictifs connus, les solutions solides suivantes ont été préparées au laboratoire et étudiées :

$$
\begin{array}{ll}
0,9\left(\mathrm{PbMg}_{1 / 3} \mathrm{Nb}_{2 / 3}\right) \mathrm{O}_{3}-0,1 \mathrm{PbTiO}_{3} & (\mathrm{PMN}-\mathrm{PT}) \\
\mathrm{Pb}_{0,9} \mathrm{La}_{0,1}\left(\mathrm{Zr}_{0,65} \mathrm{Ti}_{0,35}\right) & (\mathrm{PLZT}) \\
\left(\mathrm{Pb}_{0,73} \mathrm{Ba}_{0,27}\right)_{0,97} \mathrm{Bi}_{0,02}\left(\mathrm{Zr}_{0,7} \mathrm{Ti}_{0,3}\right) \mathrm{O}_{3} & (\text { Ba.PZT })
\end{array}
$$

Les différents coefficients électrostrictifs, les valeurs de $\varepsilon$ statique et $\varepsilon_{\mathrm{r}}$ dynamique à température ambiante, la température de Curie $T_{\mathrm{c}}$ et la constante de Curie $C$ des céramiques correspondant à ces trois familles de matériaux sont donnés dans le tableau III du paragraphe 4.4 .

\section{Méthode de mesure des déformations.}

Le principe du dispositif de mesure des déformations est donné figure 3. L'échantillon de diamètre $\phi$ et d'épaisseur $e$, voisins respectivement de 10 et $1,5 \mathrm{~mm}$ est placé sur un support constitué d'une plaque en quartz fondu dont le coefficient de dilatation linéaire thermique est très faible $\left(0,5 \times 10^{-6} /{ }^{\circ} \mathrm{C}\right)$ et le coefficient de dureté important ( 7 dans l'échelle de MOHS). Cette plaque est elle-même fixée sur une table en marbre. Deux palpeurs peuvent être positionnés successivement l'un sur l'épaisseur de la céramique, l'autre sur son diamètre. Chaque palpeur est constitué par une tige en quartz solidaire du noyau d'un transformateur différentiel qui fournit une tension de sortie dont l'amplitude correspond à la valeur de la translation et la phase au sens de déplacement. Ce signal est ensuite amplifié et redressé par un ensemble électronique Tesa ou Millitron qui délivre une tension continue proportionnelle à $\Delta e$ ou $\Delta \phi$, et dont la sortie est reliée à l'une des deux entrées d'un enregistreur $X-2 Y$. La haute tension est appliquée sur l'échantillon par l'intermédiaire de deux lames de ressort souples à l'aide d'un générateur haute tension très basse

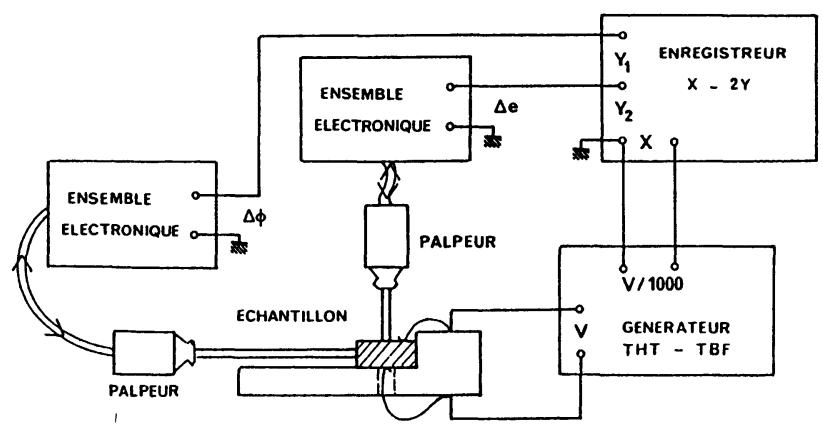

Fig. 3. - Schéma du principe du dispositif de mesure des déformations.

[Block diagram of the deformation measurement device.] 
fréquence ; ceci permet d'éviter une élévation de la température de l'échantillon par l'énergie calorifique dissipée à chaque cycle. Tous les cycles ont été tracés à la fréquence de $0,1 \mathrm{~Hz}$.

La précision des mesures est liée essentiellement à la dérive thermique; elle est de l'ordre de $5 \%$ maximum.

La figure 4 donne les enregistrements des déformations selon le mode longitudinal et le mode transverse pour le matériau de formule

$$
\mathrm{Pb}_{0,74} \mathrm{Sr}_{0,18} \mathrm{Ba}_{0,10}\left(\mathrm{Zr}_{0,54} \mathrm{Ti}_{0,46} \mathrm{Nb}_{0,02}\right) \mathrm{O}_{3}
$$

A partir de ces enregistrements, il est possible de tracer points par points les courbes donnant les déformations relatives $|\Delta \ell / \ell|$ selon les deux modes en fonction du carré du champ appliqué (Fig. 5 et suivantes). D'après les relations donnant la déformation en fonction de $E^{2}\left(|\Delta \ell / \ell|=M E^{2}\right)$ on peut calculer les coefficients $M_{11}$ et $M_{12}$ (suivant les valeurs croissantes de $E$ ) ; en calculant la valeur de $\varepsilon$ statique à partir du tracé des cycles d'hystérésis, on peut en déduire les coefficients $Q_{11}$ et $Q_{12}$ $\left(M=\varepsilon^{2} Q\right)$, donc le coefficient $Q_{\mathrm{h}}$.

\section{Résultats.}

4.1 ChOIX D'UNE SOLUTION SOLIDE $\mathrm{Pb}\left(\mathrm{Zr}_{1-x} \mathrm{Ti}_{x}\right)$ $\mathrm{O}_{3}(\mathrm{PZT})$. - Etant donné les caractéristiques retenues : grande valeur de $\varepsilon_{\mathrm{r}}$, température de Curie près de l'ambiante, cycle d'hystérésis très mince, et

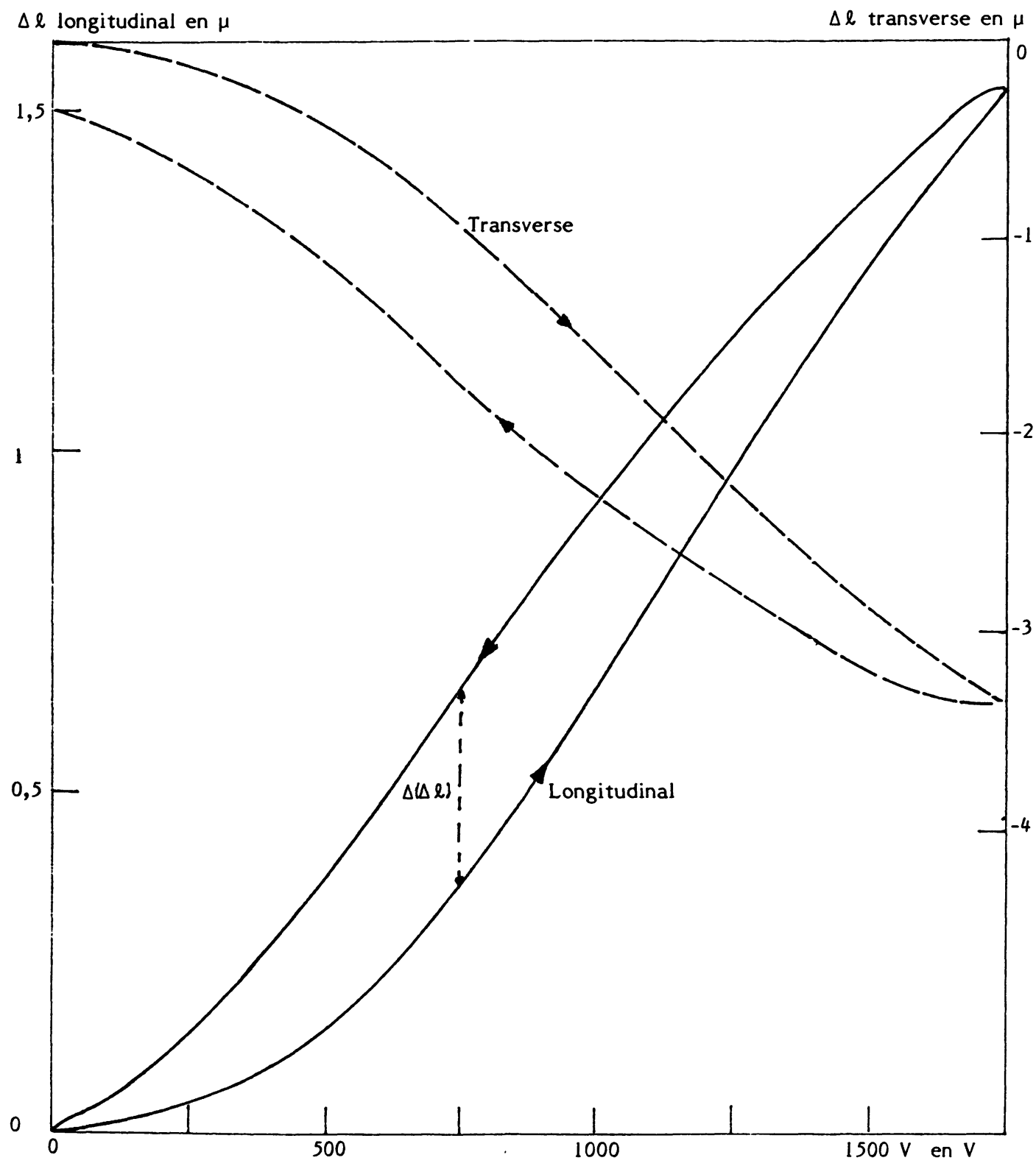

Fig. 4. - Déformations en fonction de la tension en modes longitudinal et transverse.

[Longitudinal and transverse deformation as a function of driving voltage.] 


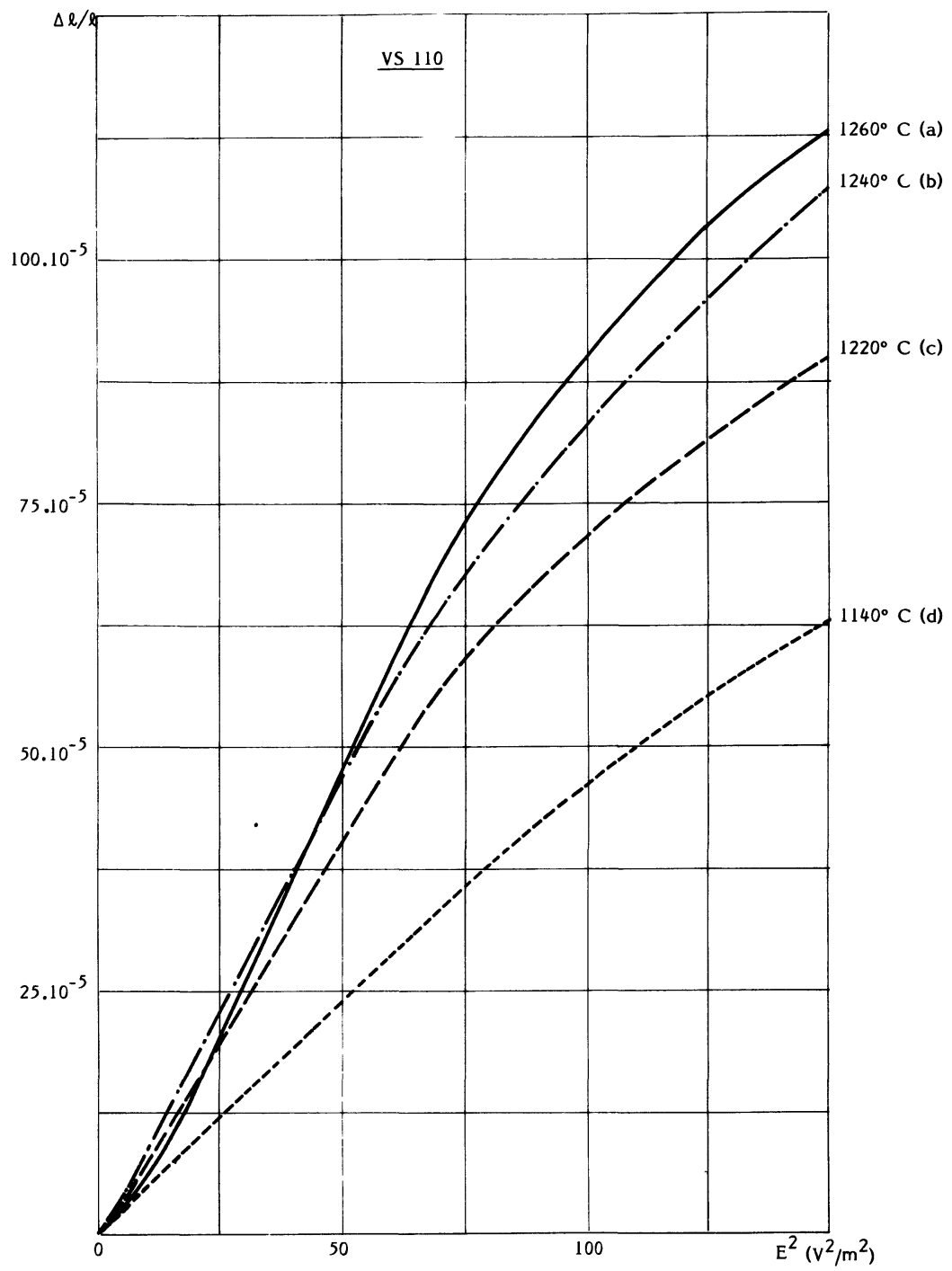

Fig. 5. - VS 110. Déformations en fonction du carré du champ pour différentes températures de frittage.

[VS 110. Strains versus electric field for various sintering temperatures.]

compte tenu des travaux antérieurs [8] faits sur les matériaux à forte substitution de $\mathrm{Pb}$ par le couple $\mathrm{Sr}$ $\mathrm{Ba}$, différents essais ont été effectués sur ce type de solution solide $\mathrm{Pb}(\mathrm{Sr}, \mathrm{Ba})(\mathrm{Zr}, \mathrm{Ti}) \mathrm{O}_{3}$.

Les matériaux ont été préparés par voie solide selon le procédé habituel résumé par le tableau synoptique 1 .

Différents essais en fonction du pourcentage Sr$\mathrm{Ba}$ et du rapport $\mathrm{Zr}$ - $\mathrm{Ti}$ ont montré que le matériau qui donnait les déformations les plus élevées en fonction du champ appliqué répondait à la composition formulaire :

$$
\mathrm{Pb}_{0,74} \mathrm{Sr}_{0,18} \mathrm{Ba}_{0,10}\left(\mathrm{Zr}_{0,54} \mathrm{Ti}_{0,46} \mathrm{Nb}_{0,02}\right) \mathrm{O}_{3}
$$

référencé VS 110.

4.2 INFLUENCE DES CONDITIONS DE FRITTAGE. L'influence de la taille des grains de la céramique sur la déformation ainsi que sur l'hystérésis de cette REVUE DE PHYSIQUE APPLIQUÉE. - T. 23, N 5, MAI 1988 déformation en fonction du champ appliqué a été mise en évidence par certains auteurs [7]. Lors de la fabrication d'une céramique, différents facteurs interviennent pour faire varier la taille des grains du matériau final, entre autres la température de frittage et le temps de frittage. Nous avons en particulier étudié sur le matériau VS 110 l'influence de la température de frittage.

A partir de la poudre obtenue après séchage nous avons fritté des céramiques à des températures allant de 1140 à $1260^{\circ} \mathrm{C}$ pendant $2,5 \mathrm{~h}$. La figure 5 donne les déformations pour 4 de ces essais et la figure 6 les photographies des grains obtenues après attaque chimique de la surface polie des céramiques. Le diamètre moyen des grains a été estimé par la méthode de la diagonale. Il apparaît clairement que les céramiques à gros grains présentent une meilleure électrostriction. Cependant, outre la déformation, un critère déterminant dans le choix des matériaux 


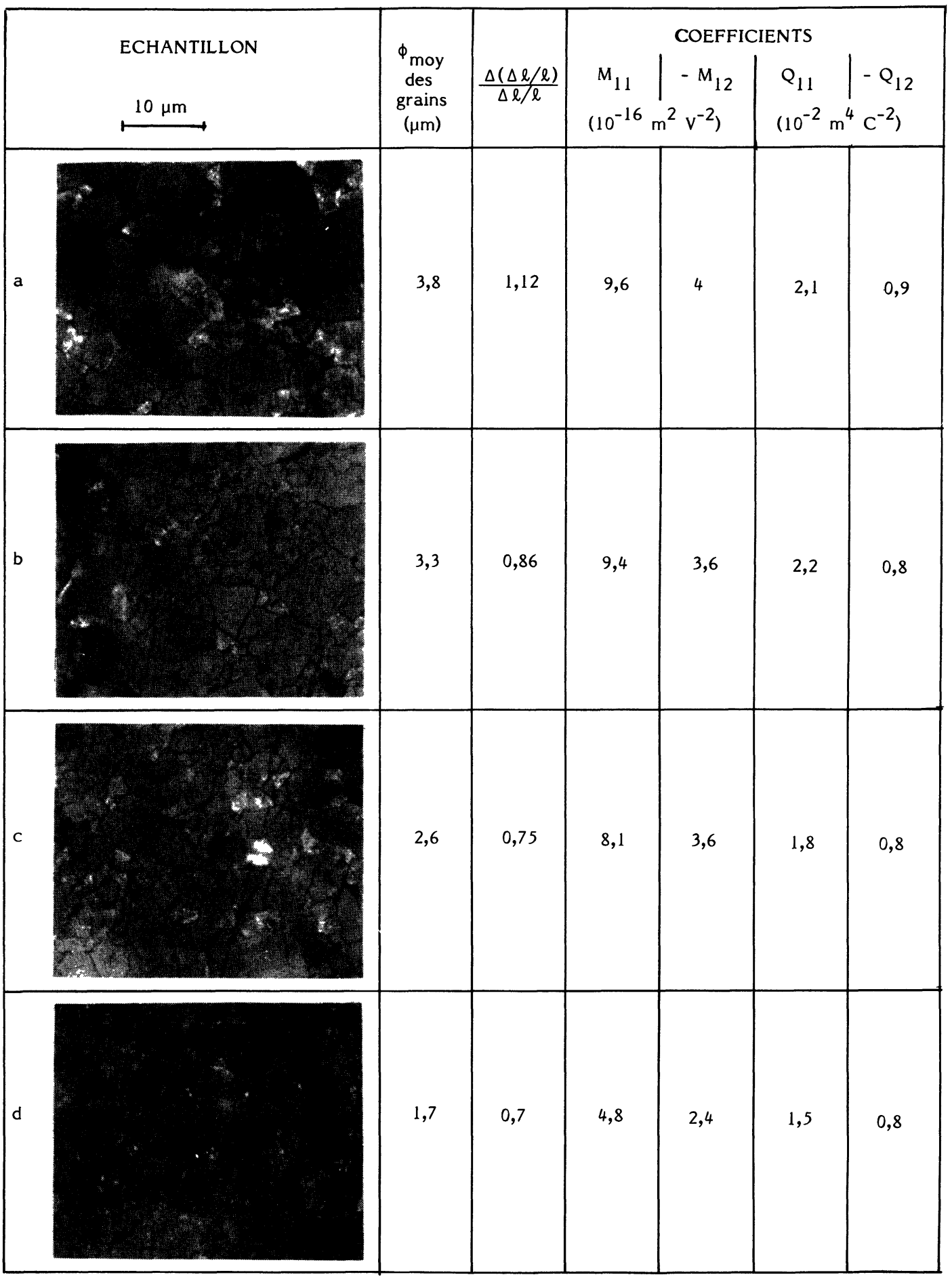

Fig. 6. - VS 110. Granulométrie des céramiques et valeurs des différents paramètres électrostrictifs suivant la température de frittage $\left(a=1260^{\circ} \mathrm{C}, b=1240{ }^{\circ} \mathrm{C}, c=1220^{\circ} \mathrm{C}, d=1140^{\circ} \mathrm{C}\right)$.

[VS 110. Ceramics grain size and electrostrictive coefficients according to the sintering temperatures.]

électrostrictifs est l'importance de l'hystérésis de déformation pendant l'application et la rupture du champ électrique. Nous avons donc mesuré sur les courbes de déformation tracées pour un champ électrique variant de 0 à $1500 \mathrm{~V} / \mathrm{mm}$ l'hystérésis longitudinale $\Delta(\Delta \ell / \ell)$ pour une valeur du champ appliqué égale à $500 \mathrm{~V} / \mathrm{mm}$ (cf. Fig. 4) et enfin fait le rapport $\Delta(\Delta \ell / \ell) / \Delta \ell / \ell$ pour cette même valeur. Les résultats obtenues pour les 4 essais retenus, ainsi que les coefficients électrostrictifs sont consignés sur la figure 6 . On observe que le matériau correspondant au frittage (b) présente un bon compromis entre une bonne valeur du coefficient $M_{11}$ et une hystérésis pas trop importante. Ces observations 
sont comparables à celles données par Heywang [9] pour $\mathrm{BaTiO}_{3}$.

\subsection{FABRICATION PAR VOIE LIQUIDE DU MATÉRIAU} $\mathrm{PZT}$ : référencé VL 779 . — La préparation des céramiques par le procédé de coprécipitation en milieu oxalique $[8,10,11]$ est résumée sur le tableau II. Nous l'avons utilisée pour la fabrication du produit précédemment mis au point par la voie solide.

Tableau II. - Tableau synoptique de la préparation des matériaux par la méthode de coprécipitation.

[Flow chart of the material preparation through the coprecipitation method.]

\section{SOLUTION AQUEUSE D'ACIDE OXALIQUE}
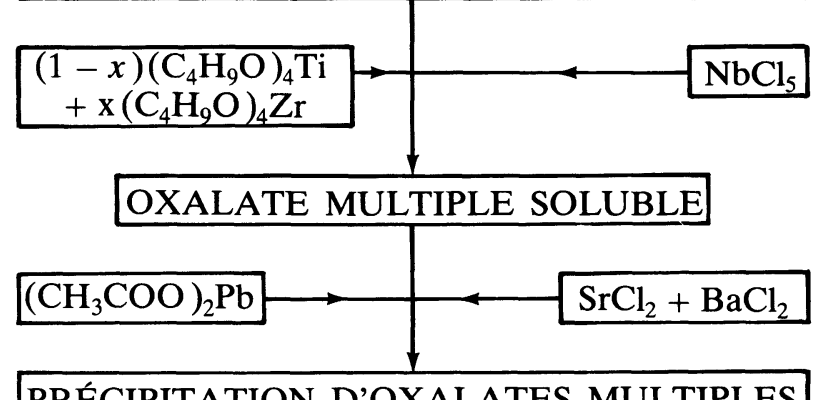

PRÉCIPITATION D'OXALATES MULTIPLES

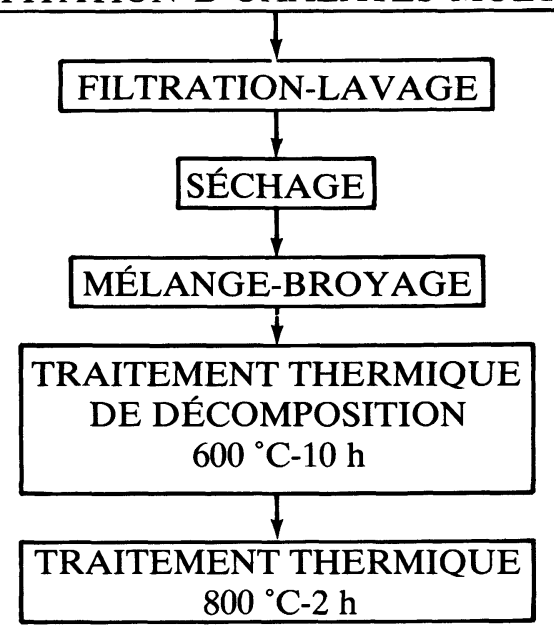

L'un des intérêts de ce procédé est de pouvoir agir par un traitement thermique approprié sur la granulométrie des poudres avant frittage, de sorte que les conditions de frittage (temps, température) ont moins d'influence sur les propriétés finales de la céramique comme le montre la figure 7 relative aux déformations obtenues pour différents frittages. Par ailleurs on peut constater sur la figure 8 que les céramiques fabriquées selon ce procédé ont par rapport au même composé préparé par voie solide, une granulométrie plus régulière et une porosité réduite, ce qui se traduira par une amélioration des coefficients d'électrostriction.

4.4 RÉCAPITUlation DES RÉSUltats. - Le tableau III donne les valeurs de quelques constantes $\left(T_{\mathrm{c}}, C, \varepsilon_{\mathrm{r}}\right.$ dynamique, $\varepsilon$ statique) et des coefficients d'électrostriction pour les céramiques PMN-PT, PLZT, Ba.PZT, préparées par voie solide et pour les céramiques (Ba-Sr).PZT, préparées par voies solide et liquide.

Les figures 9 et 10 donnent pour ces matériaux les courbes des déformations longitudinale et transverse en fonction du champ appliqué. On remarque que le matériau préparé par coprécipitation a d'excellents coefficients d'électrostriction longitudinale et transverse, de même que la composition du type PLZT qui présente en outre une hystérésis de déformation sous champ appliqué légèrement plus faible; les propriétés de ce composé devraient encore être améliorées en utilisant le procédé de préparation par voie liquide.

L'hystérésis de déformation demeure la moins élevée pour la composition $0,9 \mathrm{PMN}-0,1 \mathrm{PT}$. On peut remarquer cependant que la composition VS 110 a une hystérésis qu'il est possible de diminuer par modification des conditions de frittage tout en conservant des coefficients d'électrostriction encore appréciables (Fig. 6). Deux matériaux PLZT et Ba.PZT présentent une constante de Curie élevée et un coefficient $Q h$ voisin de 0, ce qui laisse supposer une bonne stabilité en température (cf. paragraphe 2.1).

Tableau III. - Coefficients piézoélectriques et électrostrictifs des différents matériaux électrostrictifs préparés.

[Piezoelectric and electrostrictive coefficients for the various electrostrictive samples prepared.]

\begin{tabular}{|c|c|c|c|c|c|c|c|c|c|c|}
\hline Composition & $\begin{array}{c}T_{\mathrm{c}} \\
\left({ }^{\circ} \mathrm{C}\right)\end{array}$ & $\varepsilon_{\mathrm{r}}$ ambiante & $\begin{array}{c}\varepsilon \text { statique } \\
10^{-x} \\
\text { F. } \mathrm{m}^{-1}\end{array}$ & $\begin{array}{c}C \\
10^{5}(\mathrm{~K})\end{array}$ & $\begin{array}{c}M_{11} \\
10^{-16} \\
\mathrm{~m}^{2} \mathrm{~V}^{-2}\end{array}$ & $\begin{array}{c}Q_{11} \\
10^{-2} \\
\mathrm{~m}^{4} \mathrm{C}^{-2}\end{array}$ & $\begin{array}{c}-M_{12} \\
10^{-16} \\
\mathrm{~m}^{2} \mathrm{~V}^{-2}\end{array}$ & $\begin{array}{c}-Q_{12} \\
10^{-2} \\
\mathrm{~m}^{4} \mathrm{C}^{-2}\end{array}$ & $\begin{array}{c}Q_{\mathrm{h}} \\
10^{-2} \\
\mathrm{~m}^{4} \mathrm{C}^{-2}\end{array}$ & $\frac{\left(\frac{\Delta l}{\ell}\right)}{\Delta \ell / \ell}$ \\
\hline 0,9 PMN-0,1 PT & 36 & 11500 & 23,5 & 2,1 & 9,7 & 1,7 & 2,7 & 0,5 & 0,7 & 0,7 \\
\hline $\begin{array}{l}\text { PLZT } 10 / 65 / 35 \\
\text { (Ba-Sr).PZT } \\
\text { VS } 110\end{array}$ & 84 & 4600 & 25,9 & 3,2 & 16,8 & 2,5 & 8,4 & 1,25 & 0 & 1,1 \\
\hline $\begin{array}{l}\text { voie solide } \\
\text { VL } 779\end{array}$ & 71 & 6400 & 21,3 & 2 & 9,6 & 2,1 & 4 & 0,9 & 0,3 & 1,12 \\
\hline voie liquide & 69 & 7800 & 22,6 & 2,2 & 16,5 & 3,2 & 7 & 1,4 & 0,4 & 1,5 \\
\hline Ba.PZT & 79 & 6400 & 20,2 & 3,3 & 6 & 1,5 & 3 & 0,75 & $\mathbf{0}$ & 1,9 \\
\hline
\end{tabular}




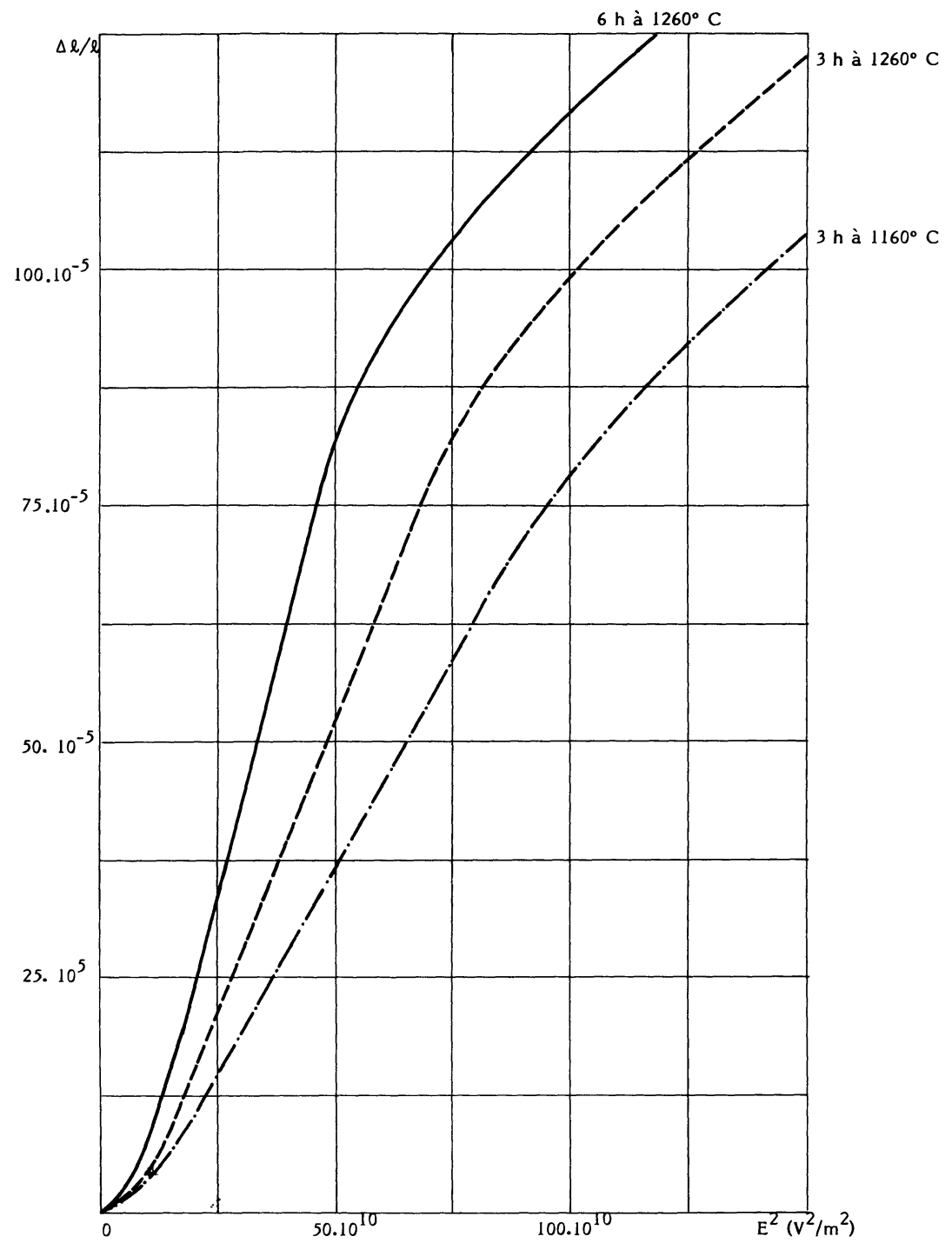

Fig. 7. - VL 779. Déformations en fonction du carré du champ pour différentes températures de frittage.

[VL 779. Strains versus electric field for various sintering temperatures.]

Pour les meilleurs matériaux piézoélectriques le coefficient piézoélectrique $d_{33}$ statique qui relie la déformation au champ électrique appliqué est de l'ordre de $850 \times 10^{-12} \mathrm{~m} / \mathrm{V}$. Si nous nous plaçons dans la partie quasi linéaire du meilleur matériau électrostrictif, soit pour un champ compris entre 500 et $750 \mathrm{~V} / \mathrm{mm}$ (Fig. 9) nous trouvons une valeur de $d_{33}$ de l'ordre de $2500 \times 10^{-12} \mathrm{~V} / \mathrm{mm}$; ce résultat montre l'intérêt du matériau électrostrictif par rapport au matériau piézoélectrique, à la condition de pouvoir appliquer au système un champ continu élevé ; cet inconvénient peut cependant être contourné par la fabrication des éléments d'actuateurs en film mince.

\section{Conclusion.}

Le matériau PMN-PT est un bon électrostrictif du point de vue hystérésis de déformation, mais sa préparation présente quelques difficultés par suite de la formation possible d'une phase pyrochlore au cours de sa fabrication [4].

Les compositions du type PLZT ou (Sr-Ba) PZT présentent d'excellentes propriétés électrostrictives, mais aux dépens de l'hystérésis. Ce dernier point peut cependant être amélioré par l'étude systématique des conditions de frittage pour contrôler la granulométrie de la. céramique (caractéristique déjà améliorée par la préparation du matériau par voie liquide). Pour certaines applications, il peut être utile de définir le meilleur compromis entre la plus grande déformation et la plus faible hystérésis.

\section{Remerciements.}

Les auteurs adressent leurs remerciements au Ministère de la Défense pour l'aide financière apportée à cette étude. 


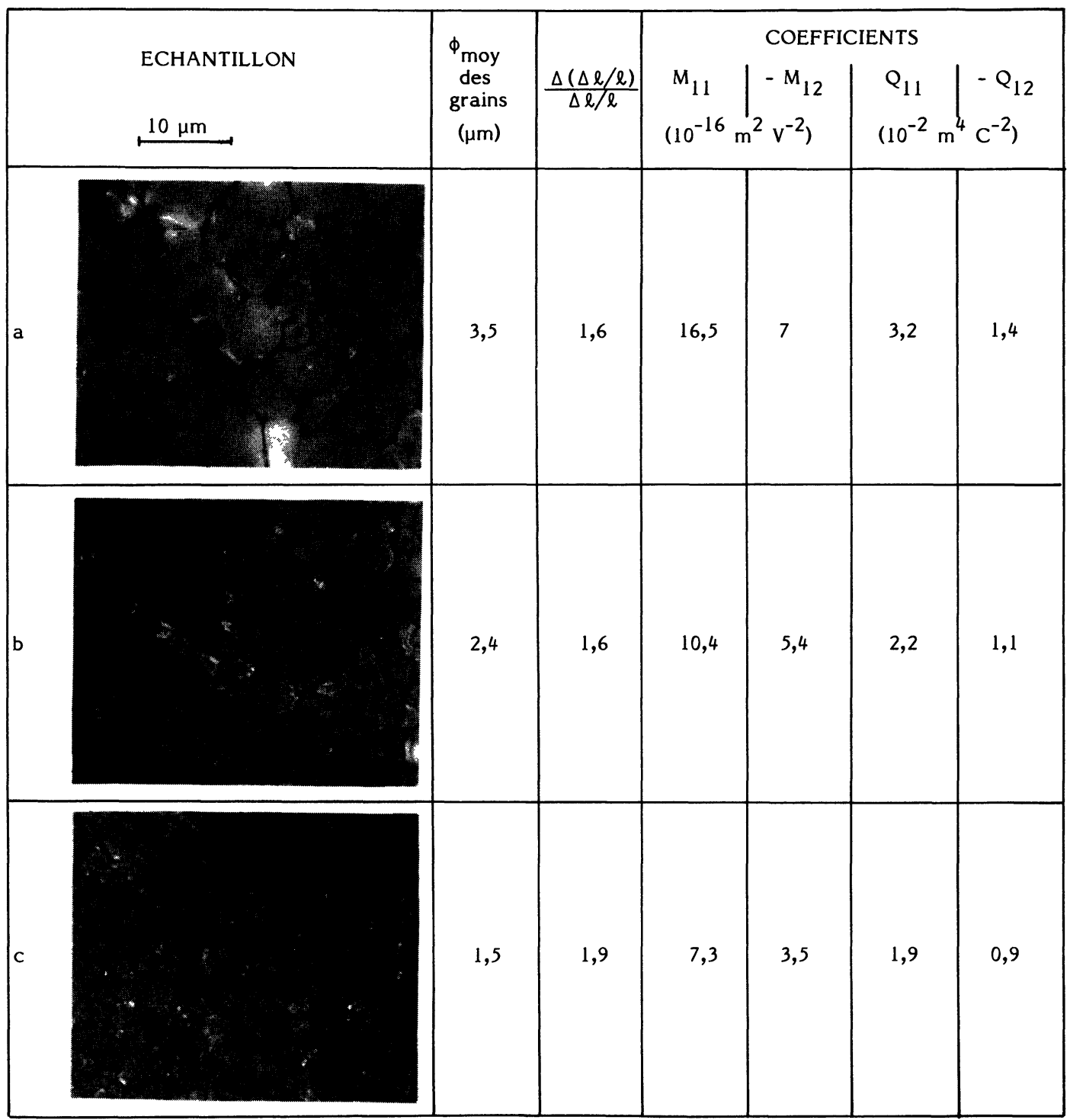

Fig. 8. - VL 779. Granulométrie des céramiques et valeurs des différents paramètres électrostrictifs suivant les conditions de frittage.

[VL 779. Ceramics grain size and electrostrictive coefficients for various sintering temperatures.] 


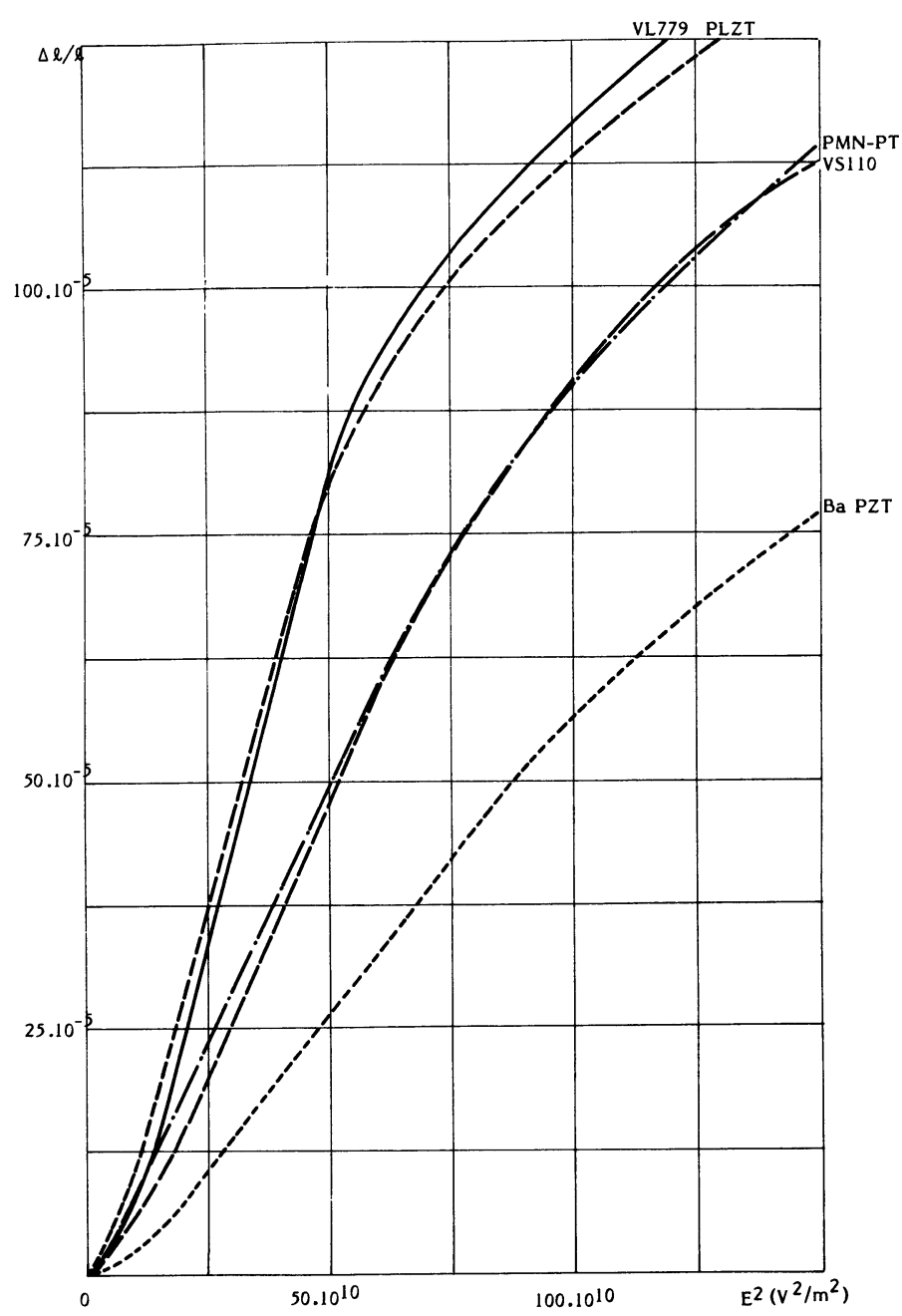

Fig. 9. - Déformations en fonction du champ pour différents matériaux électrostrictifs en mode longitudinal. [Field-induced longitudinal strains for different electrostrictive specimens.]

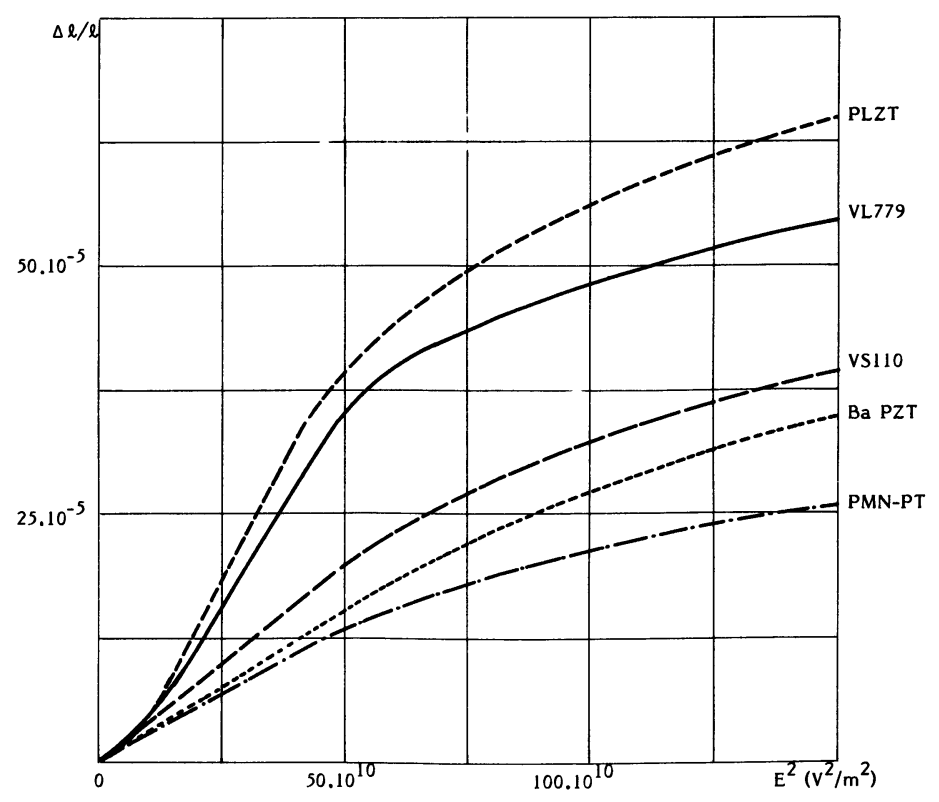

Fig. 10. - Déformations en fonction du champ pour ces mêmes matériaux en mode transverse.

[Field-induced transverse strains for the same specimens.] 


\section{Bibliographie}

[1] Kay, H. F., Rep. Prog. Phys. 43 (1955) 230.

[2] Cross, L. E., Jang, S. J., Newnham, R. E., Nomura, S. and UChino, K., Ferroelectrics 23 (1980) 187.

[3] Uchino, K., Cross, L. E. and Nomura, S., J. Mater. Sci. 15 (1980) 2643.

[4] Swartz, S. L., Shrout, T. R., Schulze, W. A. and Cross, L. E., J. Am. Ceram. Soc. 67 (1984) 311.
[5] Uchino, K., Nomura, S., Cross, L. E., Newnham, R. and JANG, S. J., J. Mater. Sci. 16 (1981) 569.

[6] Lejeune, M., Thèse d'Etat (Limoges) janvier 1986.

[7] Uchino, K., Am. Ceram. Soc. Bull. 65 (1986) 647.

[8] Eyraud, L., Eyraud, P., Gonnard, P. and TrocCAZ, M., Ferroelectrics 31 (1981) 113.

[9] Heywang, W., Ferroelectrics 49 (1983) 3.

[10] Eyraud, L., Eyraud, P., Gonnard, P. and TrocCAZ, M., Ferroelectrics 34 (1981) 133.

[11] Eyraud, L., Ind. Céram. 761 (1982) 343. 